\title{
Gab Adapter Proteins as Therapeutic Targets for Hematologic Disease
}

\author{
Sheetal Verma, ${ }^{1}$ Tamisha Vaughan, ${ }^{1}$ and Kevin D. Bunting ${ }^{1,2}$ \\ ${ }^{1}$ Aflac Cancer Center of Children's Healthcare of Atlanta, Department of Pediatrics, Emory University, Atlanta, GA 30322, USA \\ ${ }^{2}$ Aflac Cancer Center and Blood Disorders Service, Division of Hematology-Oncology, Department of Pediatrics, \\ Emory University School of Medicine, 2015 Uppergate Drive NE, ECC 444, Atlanta, GA 30322, USA
}

Correspondence should be addressed to Kevin D. Bunting, kevin.bunting@emory.edu

Received 14 June 2011; Revised 30 August 2011; Accepted 6 September 2011

Academic Editor: Cheng-Kui Qu

Copyright () 2012 Sheetal Verma et al. This is an open access article distributed under the Creative Commons Attribution License, which permits unrestricted use, distribution, and reproduction in any medium, provided the original work is properly cited.

\begin{abstract}
The Grb-2 associated binder (Gab) family of scaffolding/adaptor/docking proteins is a group of three molecules with significant roles in cytokine receptor signaling. Gabs possess structural motifs for phosphorylation-dependent receptor recruitment, Grb2 binding, and activation of downstream signaling pathways through p 85 and SHP-2. In addition, Gabs participate in hematopoiesis and regulation of immune response which can be aberrantly activated in cancer and inflammation. The multifunctionality of Gab adapters might suggest that they would be too difficult to consider as candidates for "targeted" therapy. However, the one drug/one target approach is giving way to the concept of one drug/multiple target approach since few cancers are addicted to a single signaling molecule for survival and combination drug therapies can be problematic. In this paper, we cover recent findings on Gab multi-functionality, binding partners, and their role in hematological malignancy and examine the concept of Gab-targeted therapy.
\end{abstract}

\section{Discovery and Similarities of Gab Family Members}

The Gab proteins, Gab1, Gab2, and Gab3, comprise a family of scaffolding/docking molecules involved in multiple signaling pathways mediated by receptor tyrosine kinases (RTKs) and non-RTK receptors. Gab proteins integrate and amplify signals from a wide variety of sources including growth factor, cytokine, and antigen receptors, as well as cell adhesion molecules. They are subject to complex regulation by feedforward and feedback phosphorylation events as well as protein-protein interactions. Gab proteins range from 50 to $100 \mathrm{kDa}$ in size [1] and were originally identified as the mammalian homologs of the daughter of sevenless (DOS) Drosophila adapter proteins [2, 3]. They also display sequence similarity to Suppressor of Clear 1 (Soc1), which was identified by genetic screen in C. elegans $[3,4]$.

Gab1 was originally identified as a binding protein for Grb-2 [5], and Gab2 was isolated by the purification of a binding partner for SHP [6]. The discovery of Gab3 was aided by a large sequencing project, and its isolation was based on sequence similarities to Gab1 and Gab2 [7]. Very recent entries at both the genomic DNA and transcript level have been recorded for Gab4 gene in both humans and chimpanzees, but this gene is not present in mice. The human Gab4 gene is located on chromosome 22q11.1 and its nucleotide sequence is most related to Gab2 [8].

The overall sequence homology between Gab family members is about $40-50 \%$. All Gab proteins share a similar modular structure, including a Pleckstrin homology $(\mathrm{PH})$ domain at their $\mathrm{N}$-terminus, proline-rich regions in the central part, and multiple phosphorylated tyrosine residues (Figure 1). The PH domain is an approximately 100 amino acid domain that binds phosphoinositides. Gab2 binds preferentially to the PI-3K product phosphatidylinositol-3,4,5trisphosphate (PIP3), which is only found within the plasma membrane [9]. The $\mathrm{PH}$ domain mediates recruitment of Gab2 to phagocytic cups induced by Fc $\gamma$ RI and is required for fibroblast growth factor-induced tyrosine phosphorylation of this docking protein. The $\mathrm{PH}$ domain might play an important role to localize or to concentrate Gab proteins to membrane areas where receptors are activated [2]. 


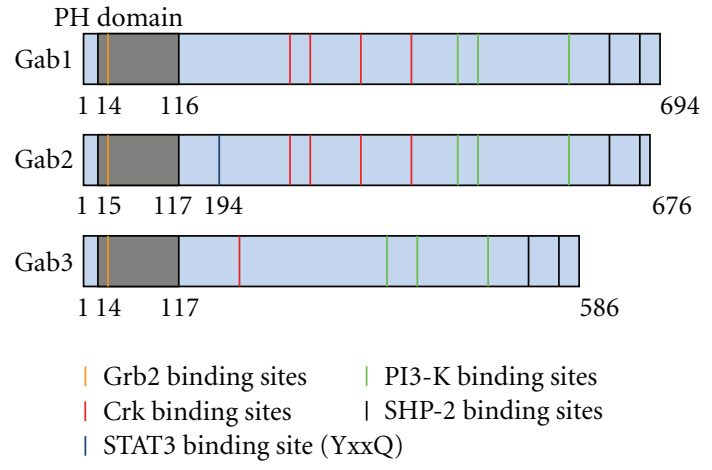

Figure 1: Gab structure and multiple binding sites. Gab molecules were originally identified as the mammalian homologs of the daughter of sevenless (DOS) drosophila adapter protein. Gab1, Gab2, and Gab3 share many common binding sites. All three Gabs have a PH domain in the amino-terminus that is believed to be essential for many functions including membrane localization. Gabs contain multiple binding sites and act as scaffolding molecules to support cytokine signaling. Binding sites for Grb2, Crk, PI3-K, and SHP-2 have been defined and extensively studied.

The phosphotyrosine domains and the proline-rich sequences (PXXP) are potential binding sites for Src homology2 (SH2) and SH3 domains, respectively. The positions of the SH2-binding tyrosine-based motifs and the SH3-binding proline-rich sequence are conserved among the Gabs. These are one of the most prominent motifs in signaling molecules due to their relevance in binding and "docking" phosphorylated tyrosine residues or directing protein-protein interaction $[10,11]$.

These adapter proteins serve important roles in cytokine receptor signaling by acting as a scaffold and coordinating interactions between signaling intermediates. Multiple protein binding motifs are present in many of the adapter molecules leading to multimeric complexes that may also include proteins such as CrkL [12], PLC- $\gamma$ [13], SHIP [14], SHP-2 [15], STAT3 [16], and STAT5 [17].

\section{Involvement in Normal and Leukemic Signaling Pathways}

As mentioned above, one of the fundamental mechanisms for regulation of Gab-mediated signal transduction is sitespecific tyrosine phosphorylation of these proteins. These molecules are involved in the phosphatidylinositol-3 kinase (PI3-K) and mitogen-associated protein kinase (MAPK) pathways and include multiple protein binding sites [18]. To further elaborate, Figure 2 illustrates how Gab2 is involved in PI-3K and MAPK pathways. These proteins are tyrosine phosphorylated following cytokine stimulation which enables interaction with a large number of partners. Table 1 summarizes a few key receptors which are associated with hematopoiesis, some of which are also found mutated in association with hematologic malignancy. Serine phosphorylation of Gabs by downstream effectors also has been described $[19,20]$, which will be discussed later.
The significance of the phosphorylation sites present on Gabs not only lies in aiding its interaction with crucial binding partners, but also on how it influences activation of downstream cytokine receptors. Interaction between the protein tyrosine phosphatase Shp2 and Gab2 regulates MAPK pathway activation. Notably, mast cells and macrophages from Gab2 $2^{-1-}$ mice have decreased Erk activation in response to SCF [27]. Gab2-Shp2 complex also appears to have an additional, distinct signaling role in response to other stimuli. For example, overexpression of Gab2 ${ }^{\mathrm{Y} 604 \mathrm{~F} / \mathrm{Y} 633 \mathrm{~F}}$ mutant fails to bind to Shp2 and blocks IL-3evoked gene activation [6]. Further, the Gab/Shp2 complex also positively regulates other downstream pathways. These include c-Kit-induced Rac activation [28], where Gab2 via Shp-2 transmitted signals from Kit receptor (Tyr-567) to activate the Rac/JNK pathway. This in turn is significant for mast cell development [28]. Another example of downstream regulation is the Gab2-mediated PI3K activation wherein, this activation is necessary for epidermal growth factor(EGF-) induced DNA synthesis in rat hepatocytes $[58,59]$.

It is noteworthy that persistent activation of c-Kit and c$\mathrm{Mpl}$ induces hematological malignancies. Activation of PI$3 \mathrm{~K}$ by c-Kit is dependent both on the direct PI3K-binding site in c-Kit and on the phosphorylation of Gab2 [54]. The fact that c-Kit has been found mutated in numerous human malignancies, including acute myeloid leukemia, and that Gab2 is often overexpressed in acute myeloid leukemia suggests a potential role of Gab2-mediated PI3K activation in transformation [60]. TPO-induced stimulation of c-Mpl has been implicated in maintaining HSC quiescence and also in myeloproliferative disorders (MPDs) and Gabs play a role in regulating $\mathrm{PI}-3 \mathrm{~K}$ and MAPK pathways, in $\mathrm{c}-\mathrm{Mpl} / \mathrm{TPO}$ signaling $[55,61]$.

\section{Functional Role in Hematopoiesis Defined by Knockout Mice}

Given their integral role in cytokine signaling, it was proposed that Gabs may play important roles in hematopoiesis. However, to date, very little is known about how multiple Gabs regulate hematopoietic cytokine signaling.

Gab1 deficiency results in embryonic lethality due to developmental defects in heart, placenta, skin, and a reduced ratio of liver to body weight at E14.5 [62, 63]. Also associated with these defects was reduced Erk activation in embryonic fibroblasts in response to stimulation with platelet-derived growth factor (PDGF), epidermal growth factor (EGF), and hepatocyte growth factor (HGF). These defects were initially observed to be similar to mice lacking expression of MET receptor, HGF, PDGF, and EGF growth factors with phenotypes such as open eye lids [64], abnormal hair follicles [65], hemorrhage and cardiac hypoplasia [66], and abnormal placenta [67-69]. Later generation of SHP-2 mutant mice revealed yet again similar defects [70] indicating an essential central role for Gab1/SHP-2 interactions in mediating growth factor activation of the Erk MAP kinase pathway. More recent conditional deletion of Gab1 led to deficient Erk signaling which allowed increased insulin receptor substrate 


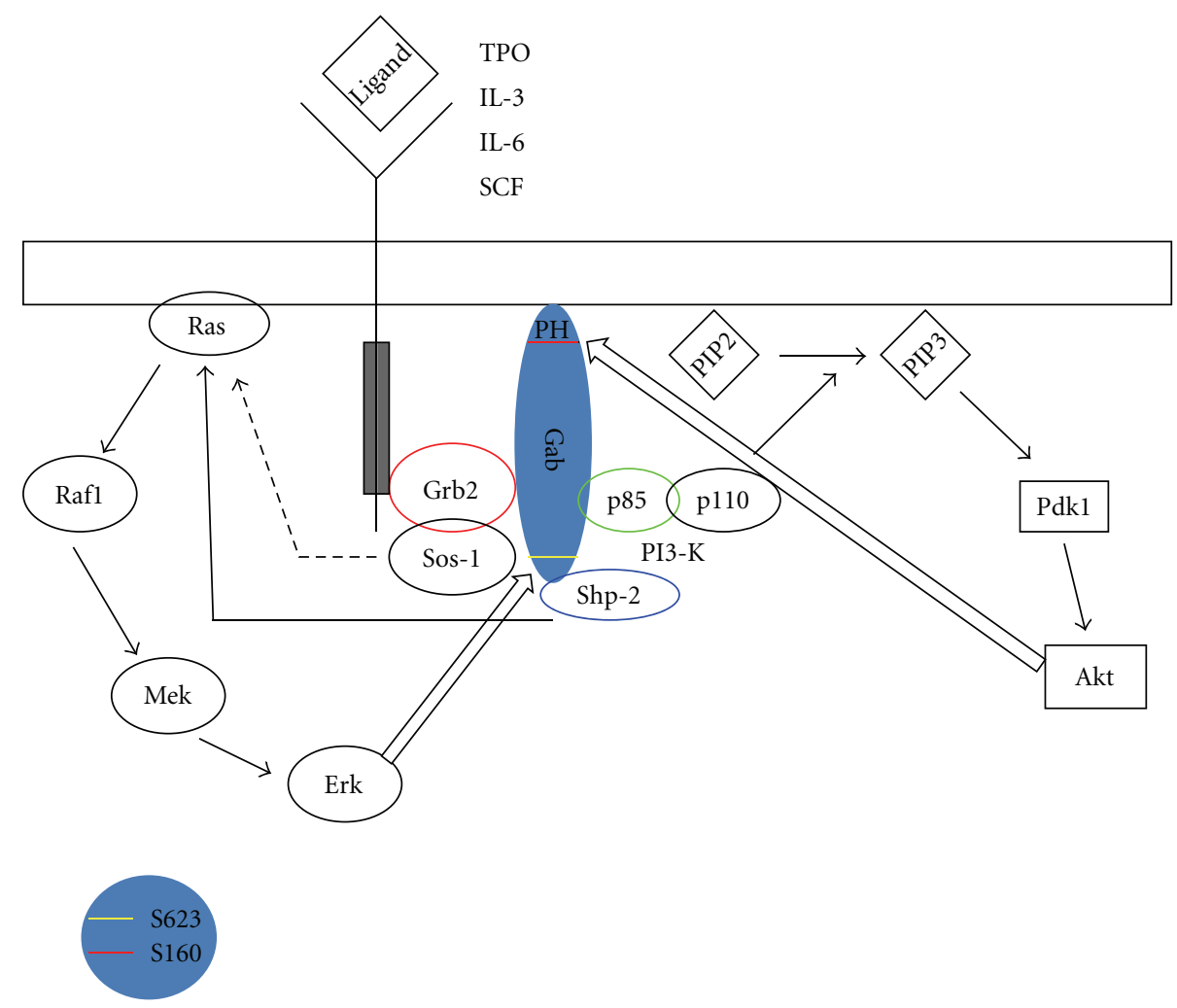

Figure 2: Gab2 interactions with binding partners. Diagram shows generic Gab adapter docked at the plasma membrane by the PH domain. Interactions with p85 and SHP-2 are involved in Akt and Erk activation respectively. Binding of receptor tyrosine kinases to their receptive ligands triggers the kinase activity of the cytoplasmic domain of the receptor. The receptor becomes phosphorylated on tyrosine residues. Docking proteins such as Grb2 contain SH2 domains that bind to the phosphotyrosine residues of the activated receptor. Grb2-Gab interacts through the SH3 domains and activates downstream signaling pathways, PI-3K/Akt and SHP2/Erk. Grb-2 can bind to SOS via N-terminal SH3 domain while the C-terminal SH3 domains are used for its interaction with Gab proteins. SOS-1 has been known to associate with Grb2, leading to its autophosphorylation. This complex gets translocated to the activated receptor where it then associates with Ras. As Ras gets activated it induces the downstream ERK/MAPK pathway. Alternatively, stimuli from growth factors like EGF, VEGF, and so forth, causes binding of Gab2 to Grb2. This then leads to recruitment of SHP2, that binds to phosphorylated tyrosine residues on Gab, and in turn activates ERK/MAPK signaling. Negative feedback by serine phosphorylation of Gab at S160 by Akt and S623 by Erk (block arrows) plays an important role in control of function and signaling of Gabs.

(IRS) activation to enhance glucose tolerance and improve hepatic insulin action [71]. A role for Gab1 as an adapter protein linking gp130 signaling to the Erk pathway has also been described [39]. Gab1 ${ }^{-/-}$cells are defective in response to gp130 activation through IL-6 and the soluble IL-6R $\alpha$ [62].

Gab2 is tyrosine phosphorylated at any of 19 sites by several early-acting cytokine receptors such as Flt3, c-Kit, IL-3R, and c-Mpl. Gab2 contains SH2 domain binding sites and 5 PxxP sites (polyProline sites) that bind $\mathrm{SH} 3$ domain $[6,18,29]$. Consistent with binding of p85 and SHP-2, Gab2 activates the PI3-K and the MAPK pathways, respectively, which may participate in regulating hematopoietic cell migration functions [48]. Gab2 $2^{-/-}$mice are viable but lack allergic response [72] since Gab2 deficiency has also been shown to alter mast cell development [27] in a manner similar to STAT5-deficient mice [73]. Use of point-mutants $[36,48,74]$, that deter Gab2 binding to signaling partners, could help to dissect the structure-function relationship of
Gab2 in vivo. A recent study has established Gab2 $\triangle \mathrm{SHP} 2$ (Y603F/Y632F) and Gab2 $\Delta \mathrm{PI} 3 \mathrm{~K}$ (Y441F/Y465F/Y573F) mutant knockin mice [75], with Gab2 mutated at SHP-2 and PI3K binding sites, respectively. Further assessment of these mice has shown that the PI3K or SHP2 binding sites in Gab2 are important for mast cell degranulation and the anaphylaxis response.

Despite the normal appearance, normal BM cellularity, and normal blood counts of mice lacking Gab2 expression, Gab2 ${ }^{-/}$mice show reduced colony forming ability in methylcellulose and impaired KLS (c-Kit ${ }^{+} \mathrm{Lin}^{-} \mathrm{Sca}-1^{+}$cell surface markers denoting mouse hematopoietic stem cell) cell growth in liquid culture [76]. The defects of these cells in response to early-acting cytokines like SCF, TPO, and IL-3 suggest that Gab2 may act as an intermediate relay protein that organizes signaling complexes and amplifies receptor activation. Owing to these findings, Gab2 can be a potential target molecule for better understanding steady-state and aberrant hematology. 
TABLE 1: Gabs are associated with multiple common hematopoietic receptors, RTK: receptor tyrosine kinase.

\begin{tabular}{|c|c|c|c|c|c|c|}
\hline \multirow[t]{2}{*}{ Receptor } & \multirow[t]{2}{*}{ Ligand } & \multirow[t]{2}{*}{ Cell type } & \multicolumn{3}{|c|}{ Phosphorylated } & \multirow[t]{2}{*}{ References } \\
\hline & & & Gab1 & Gab2 & Gab3 & \\
\hline \multicolumn{7}{|l|}{ RTK } \\
\hline Flt3 & Flt3L & BaF3/Flt3, THP-1, RS4; 11 & Yes & Yes & Yes & {$[7,21]$} \\
\hline Fms & M-CSF & $\begin{array}{l}\text { FDFms, 32D-Fms, EML-Fms, BAC1.2F5, 32D, } \\
\text { BMDM }\end{array}$ & NR & Yes & Yes & {$[6,7,22-26]$} \\
\hline Kit & SCF & FDFms, MO7E, MC9, BMMC & Yes & Yes & NR & {$[23,27-30]$} \\
\hline \multicolumn{7}{|l|}{ Non-RTK } \\
\hline EPO receptor & EPO & $\begin{array}{l}\text { BaF3, UT-7, HCD- } 57 \text {, primary erythroid cells, } \\
\text { R1, Namalwa, FDC-P1 }\end{array}$ & Yes & Yes & NR & {$[13,31-34]$} \\
\hline G-CSF receptor & G-CSF & BaF3, DT40 & NR & Yes & NR & {$[35]$} \\
\hline GM-CSF receptor & GM-CSF & FDFms, BaF3, UT-7 & Yes & Yes & NR & {$[23,36-38]$} \\
\hline gp130 & IL-6, LIF & $\begin{array}{l}\text { HepG2, BAF-B03, T47D, MM.1S, cardiomyo- } \\
\text { cytes }\end{array}$ & Yes & Yes & NR & {$[29,39-43]$} \\
\hline IL-15 receptor & IL-15 & T cells, MyLa2059 & NR & Yes & NR & {$[44,45]$} \\
\hline IL-2 receptor & IL-2 & $\begin{array}{l}\text { Kit225, KT-3, T-cell blasts, T cells, NK3.3, } \\
\text { MyLa2059 }\end{array}$ & NR & Yes & NR & {$[18,19,29,45-47]$} \\
\hline IL-3 receptor & IL-3 & $\begin{array}{l}\text { BaF3, BAF-B03, primary hematopoietic cells, } \\
\text { NIH 3T3 }\end{array}$ & Yes & Yes & Yes & {$[29,48-53]$} \\
\hline $\mathrm{Mpl}$ & $\mathrm{TPO}$ & $\begin{array}{l}\text { TF-1, UT-7, BaF3, primary megakaryocyte pro- } \\
\text { genitors }\end{array}$ & Yes & Yes & NR & {$[54-57]$} \\
\hline
\end{tabular}

NR: not reported.

In contrast to Gab1 and Gab2 which have ubiquitous expression in brain, kidney, lung, heart, and ovary $[5,6$, 9], Gab3 is localized to hematopoietic tissues [7, 77]. An additional difference between Gab3 and Gab1/2 is that it may not be able to interact with Crk or Crkl but has unique potential binding sites that have yet to be characterized [7]. Gab3 SH3 domains have been shown to associate with a number of Src family kinases including Src, Fyn, and Lyn [7]. Increased Gab3 expression is observed following MCSF stimulation of myeloid and macrophage cell lines [7], and differentiation is facilitated by overexpression. However, Gab3 knockout mice do not have obvious hematopoietic phenotypes [78] and have normal macrophage numbers.

\section{Supporting Role for Gabs in Cancer Progression}

It is well established that Gab proteins promote tumorgenesis by functioning as "accomplices" of certain oncoproteins or by amplifying signaling upon their overexpression. This type of "nononcogene addiction" has been described for molecules that become essential in the setting of cancer, but they are not mutated or capable of transformation on their own. In addition to normal cytokine activation, Gab1 and Gab2 can also be activated by oncogenic tyrosine kinases, oncoproteins, and Src family kinases (summarized in Table 2). Gab3 has not yet been described to play a role in cancer signaling.

The study of Gabl in Met signaling and cancer has been researched in recent years. Overexpression of Gab1 promotes cell cycle progression when Met is expressed at
TABLE 2: Gab1 and Gab2 are activated by kinases, oncoproteins, and other adaptors in cancer cells.

\begin{tabular}{lccc}
\hline Cancer & Gab activator & Gab1 & Gab2 \\
\hline Breast [79-83] & $\begin{array}{c}\text { Amplification, } \\
\text { ErbB2, Src }\end{array}$ & Yes & Yes \\
Glioblastoma [84] & Met & Yes & No \\
$\begin{array}{l}\text { Thyroid [85] } \\
\text { Gastric [86] }\end{array}$ & Amplification & No & Yes \\
$\begin{array}{lcc}\text { Myeloma [40] } \\
\text { Chronic myelogenous leukemia }\end{array}$ & Hck & Yes & Yes \\
$\begin{array}{l}\text { [53, 87] } \\
\text { Friend virus erythroblastosis }\end{array}$ & No & Yes \\
$\begin{array}{l}\text { 16, 88] } \\
\text { Anaplastic large cell lymphoma [89] }\end{array}$ & NPM-Alk & No & Yes \\
$\begin{array}{l}\text { Acute myeloid leukemia [90, 91] } \\
\text { Myeloproliferative disease [92] }\end{array}$ & Flt3-ITD & Yes & Yes \\
\hline
\end{tabular}

suboptimal levels. For this response, it is required for Gab1 to possess an intact Met-binding motif, the $\mathrm{PH}$ domain, and the binding sites for PI3-K and SHP-2. In this model, Gab1 sufficiently promoted transformation and proliferation of fibroblasts [93]. A role for Gab1/Shp-2 interaction in growth and transformation of NIH 3T3 fibroblasts has also been reported [94], although this has not yet been validated in vivo for disease induction. It was shown in recent studies that Gab1 expression increased cell motility and adhesion of myeloid 32D cells in a hepatocyte growth factor (HGF) stimulated setting. In this setting, Gabl was also seen to up 
regulate GATA-2, which has been implicated in CML and could be a key player in malignant transformation [95].

Involvement of Gab2 in leukemogenesis was highlighted when myeloid progenitors from Gab2-deficient mice were found to be resistant to transformation by Bcr-Abl [87]. Phosphorylation of Y177 within the Bcr moiety leads to recruitment of the Grb2/Gab2 complex and triggers downstream signaling via SHP2 and PI-3K, which is crucial for enhanced proliferation and survival. Similarly, the oncogenic Bcr-FGFR1 fusion protein, which is also the product of a chromosomal translocation, drives the tyrosine phosphorylation of Gab2 in murine bone marrow cells and their malignant transformation through phospho-Y177 mediated Grb2 association [96]. Another kinase implicated as a key component of the Bcr-Abl signaling network is Jak2 that in turn activates Lyn leading to Gab2 phosphorylation. These findings highlight the role of Gab2 phosphorylation in driving chronic myeloid leukemia (CML) [97]. After the pivotal role of Gab2 in Bcr-Abl-mediated transformation had been established, its involvement in the pathogenesis of several other leukemias was discovered. The oncogenic fusion kinases Tel-Abl and Tel-Jak2 engage Gab2 in a similar manner to Bcr-Abl [98, 99]. Likewise, it was seen that introduction of a germline gain-of-function SHP2 mutation, D61G/+, induced MPD by aberrant activation of HSCs and the disease phenotype was improved in the Ptpn11(D61G/+)/Gab2(-/-) double mutant mice [100]. This further illustrates that interactions between Gab2 and partners like SHP2 is critical for development of MPD, in vivo. However, the relative contribution of the Gab2/SHP2 interaction for Erk activation versus the reciprocal inactivation of STAT5 is highly complex and difficult to discern $[21,90,91,100]$. Both Erk and STAT5 can drive myeloproliferation, and the degree to which they cooperate in normal and leukemic hematopoiesis is not well defined.

It should be pointed out that although the focus of this review is on Gabs as interaction partners of oncoproteins involved in the transformation of hematopoietic cells, the most thoroughly described roles have been in solid tumors. Gab2 amplification has been seen in nonhematopoietic cancers such as breast cancer cell lines [79]. Gab2-mediated activation of the Shp2/Erk signaling pathway is important for the proliferation of mammary cells. Amplification of Gab2containing region has been reported in $10-15 \%$ of human breast tumors [80]. Further supporting this is the in vitro study whereby overexpression of Gab2 in human MCF-10A cells using a retroviral vector approach [81] gave similar results. Furthermore, deletion of Gab2 delays migration of mouse mammary tumors generated using breast cancer cell lines and this defect can be fully restored by reintroduction of a plasmid expressing Gab2 [82]. A particular role for Gab2 and Akt activity has been shown following E2F1 hyperactivation in p27-deficient cells leading to enhanced cell migration and invasion [101]. A recent study in MCF10A epithelial cells show Gab2 overexpression enhances cell migration and reduces formation of epithelial colonies. Further, modulation of focal adhesions by Gab2 was dependent on Shp-2 binding sites. Shp-2 binding defect mutant restored normal cell spreading. In contrast, the Shp- 2 affinity mutant promoted Vav2 phosphorylation and recruitment of some important RhoA family regulators leading to increased cell motility $[80,102,103]$.

\section{Regulation by Posttranslational Modifications}

The key to figuring out the role of Gabs in hematological disease is to understand their role in signaling cascades. It is crucial to visualize these as intertwined loops. Firstly, phosphorylation of a particular residue might affect the phosphorylation of a nearby residue in either a positive or antagonistic fashion, due to phosphorylation-induced changes in protein conformation. Secondly, phosphorylation-induced conformational changes may alter the accessibility of key regions, such as the $\mathrm{PH}$ domain.

Negative feedback regulation of Gab2 can be achieved by serine phosphorylation at sites near the $\mathrm{PH}$ domain (Akt) or near the SHP-2 binding domain (Erk). Akt can constitutively associate with Gab2, phosphorylate it on a consensus phosphorylation site (Ser159) and inhibit Gab2 tyrosine phosphorylation [20]. Mutation of S159A (corresponding mutation in mouse is S160) resulted in increased tyrosine phosphorylation of Gab2 and the Gab2 ${ }^{\mathrm{S} 159 \mathrm{~A}}$ mutant displayed transforming properties in fibroblasts and prolonged signaling through the PI-3K/Akt pathway. This might impact downstream STAT5 activity, as it has been shown that constitutively active STAT5 forms a complex with the p85 subunit of the PI3-K and Gab2 in leukemic bone marrow cells, resulting in the activation of Akt [17]. Gab2 is also regulated by Erk-mediated negative feedback phosphorylation, wherein the identified $\mathrm{S} 623$ is the site of phosphorylation of Gab2 by Erk. The Gab2/Shp2 interaction is enhanced by S623A mutation. This is in turn expected to strongly inhibit downstream STAT5 [19]. Thus, it will be of relevance to study the balance between positive and negative signaling through both in vitro models and further complement it by in vivo analysis. Negative feedback is deemed critical in shifting Gab2 signaling from Erk and Akt to STAT5. This mechanism may be particularly relevant during conditions of hematopoietic stress such as recovery from myelosuppression or leukemic hematopoiesis. These conditions are more likely to increase Gab2-mediated Erk and Akt signaling, while inhibiting STAT5, whereas blockage or complete deficiency of Gab2 might be expected to have a reverse effect (Figure 3).

\section{Targeted Therapy of Gabs?}

Since Gabs bind many common receptors and are much less studied in hematopoiesis, it is pivotal to understand the role of binding to their partners and how it impacts oncogenic tyrosine kinase signaling. Due to the fact that all three family members are potential players in various signaling pathways, it will be interesting to see the shift in approach for targeting the Gab family. As discussed earlier, total knockdown of these proteins has varied physiological and phenotypic impacts. But, it cannot be emphasized enough that the Gab 


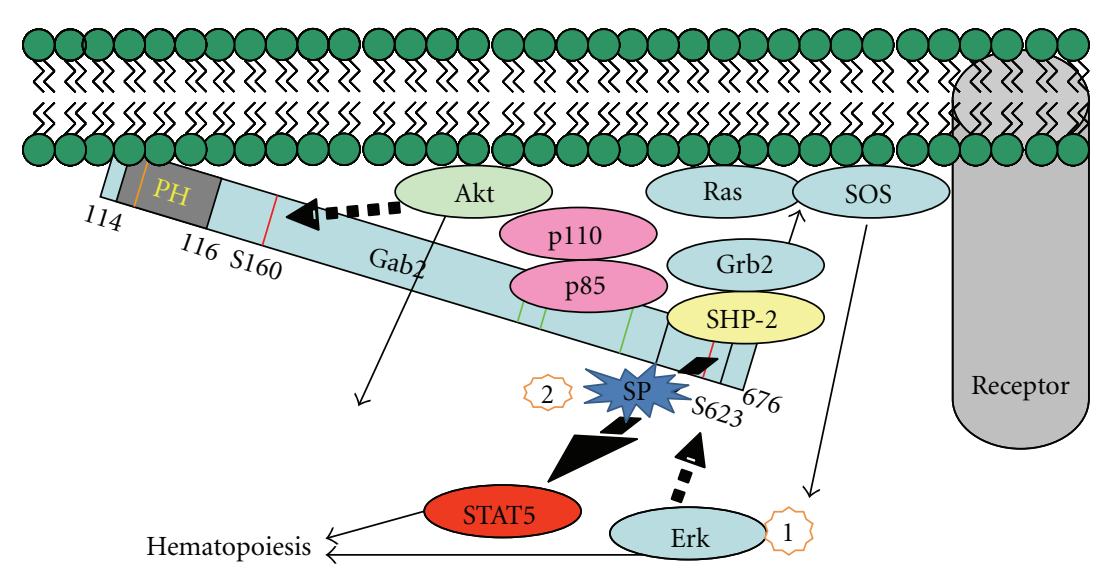

p 85 binding sites Y452/Y476/Y584
I SHP-2 binding sites Y614/Y643

FIgure 3: Potential of stapled peptides in therapeutic intervention. Step 1 illustrates how Erk phosphorylates Gab2 on a consensus phosphorylation site at serine 623, a residue located between tyrosine 614 and tyrosine 643, which are responsible for Gab2/Src homology 2 domain-containing tyrosine phosphatase- (SHP-) 2 interaction. As is reported in the text, this is part of a negative feedback loop. Hypothetically a stapled peptide (SP), a biosynthetic molecule binding to a directed region on Gab2, could be introduced in Step 2 to block the negative regulation by Erk to keep SHP-2 active and STAT5 inactive. Alternatively, it may be necessary to block SHP-2 binding to Gab2 depending on the disease entity. This figure elaborates how targeted therapy might provide new direction into understanding the interactions between adapters, like Gabs, and their partners, and which could ultimately be applied as leukemia therapy.

family members are also key regulators/enhancers of other oncogenic proteins and could compensate for the deficit of a family member.

Synthetic peptides that specifically bind to directed targets offer an approach for the modulation of receptor signaling and subsequent gene expression. Stabilized $\alpha$-helix, $\mathrm{SAH}$, is a stapled peptide to p53 that has been shown to prevent p53-MDM4 binding, enabling activation of p53 response and tumor growth suppression both in vitro and in vivo models [104]. Recently, engineered photoreactive stapled $\mathrm{BH} 3$ peptide helices covalently trap both static and dynamic protein interactors, and enable rapid identification of interaction sites, providing a new scope for targeted drug design for the BCL-2 family targets [105]. Visualizing potential sites on Gabs for similar stapled peptides can provide fascinating insights into protein-binding disruption. These disruptions could also uncover the role of binding partners in disease regulation (Figure 3 ). In some ways these directed peptides could be more practical as targeted therapy, since they would not silence the entire molecule, like siRNA targeting, but rather, could disturb binding and interactions of the target with regulators. However, these interactions are complex and as mentioned earlier both Erk and STAT5 are important for leukemogenesis, so it remains unclear whether promoting Gab2/SHP2 interaction to reduce STAT5 activation would be advantageous. The disadvantage of such a targeted therapy approach would be increased Erk activation. The therapeutic potential could depend on the particular upstream activating mutation and the stage of disease development. The use of new knockin animal models expressing mutant Gabs would be needed to address these important issues.
An alternative approach to targeting the specific effectors of Gab functional interactions would be to target the entire molecule as a whole. The risk of this approach is that it would be predicted to have widespread effects on Erk and AKT activation, as well as loss of potential negative regulatory functions. The total Gab targeting approach is also complicated by the potential redundancy of Gab1, Gab2, and Gab3 for specific Gab signaling functions. Only through rational dissection of Gab structure-function as related to disease progression will targeted therapy for Gabs become more rationally guided. It is appealing to consider adapter proteins as drug targets from the respect that they have the ability to impact upon multiple key oncogenic signals which may be cooperative. This "killing a flock of birds with one stone" may be optimal over even "killing two birds with one stone," and only adapter proteins permit widespread impact on receptor-signaling molecule interactions. Therefore, Gabs are of interest in the field of targeted therapeutics and their complete deletion shows therapeutic potential in mouse models; however, drug development for these targets will require moving forward with caution and greater understanding of structure-function relationships.

\section{References}

[1] H. Gu and B. G. Neel, "The "Gab" in signal transduction," Trends in Cell Biology, vol. 13, no. 3, pp. 122-130, 2003.

[2] T. Raabe, J. Riesgo-Escovar, X. Liu et al., "DOS, a novel pleckstrin homology domain-containing protein required for signal transduction between sevenless and Ras1 in drosophila," Cell, vol. 85, no. 6, pp. 911-920, 1996. 
[3] R. Herbst, P. M. Carroll, J. D. Allard, J. Schilling, T. Raabe, and M. A. Simon, "Daughter of sevenless is a substrate of the phosphotyrosine phosphatase corkscrew and functions during sevenless signaling," Cell, vol. 85, no. 6, pp. 899-909, 1996.

[4] J. L. Schutzman, C. Z. Borland, J. C. Newman, M. K. Robinson, M. Kokel, and M. J. Stern, "The caenorhabditis elegans EGL-15 signaling pathway implicates a DOS-like multisubstrate adaptor protein in fibroblast growth factor signal transduction," Molecular and Cellular Biology, vol. 21, no. 23, pp. 8104-8116, 2001.

[5] M. Holgado-Madruga, D. R. Emlet, D. K. Moscatello, A. K. Godwin, and A. J. Wong, "A Grb2-associated docking protein in EGF- and insulin-receptor signalling," Nature, vol. 379, no. 6565, pp. 560-564, 1996.

[6] H. Gu, J. C. Pratt, S. J. Burakoff, and B. G. Neel, “Cloning of p97/Gab2, the major SHP2-binding protein in hematopoietic cells, reveals a novel pathway for cytokine-induced gene activation," Molecular Cell, vol. 2, no. 6, pp. 729-740, 1998.

[7] I. Wolf, B. J. Jenkins, Y. Liu et al., "Gab3, a new DOS/Gab family member, facilitates macrophage differentiation," Molecular and Cellular Biology, vol. 22, no. 1, pp. 231244, 2002.

[8] F. U. Wohrle, R. J. Daly, and T. Brummer, "Function, regulation and pathological roles of the Gab/DOS docking proteins," Cell Communication and Signaling, vol. 7, p. 22, 2009.

[9] C. Zhao, D. H. Yu, R. Shen, and G. S. Feng, "Gab2, a new pleckstrin homology domain-containing adapter protein, acts to uncouple signaling from ERK kinase to Elk-1," The Journal of Biological Chemistry, vol. 274, no. 28, pp. 1964919654, 1999.

[10] Y. Liu and L. R. Rohrschneider, “The gift of Gab," Febs Letters, vol. 515, no. 1-3, pp. 1-7, 2002.

[11] B. G. Neel, H. Gu, and L. Pao, "The "Shp"ing news: SH2 domain-containing tyrosine phosphatases in cell signaling," Trends in Biochemical Sciences, vol. 28, no. 6, pp. 284-293, 2003.

[12] D. Sakkab, M. Lewitzky, G. Posern et al., "Signaling of hepatocyte growth factor/scatter factor (HGF) to the small GTPase Rap1 via the large docking protein Gab1 and the adapter protein CRKL," The Journal of Biological Chemistry, vol. 275, no. 15, pp. 10772-10778, 2000.

[13] C. Boudot, Z. Kadri, E. Petitfrère et al., "Phosphatidylinositol 3-kinase regulates glycosylphosphatidylinositol hydrolysis through PLC- $\gamma 2$ activation in erythropoietin-stimulated cells," Cellular Signalling, vol. 14, no. 10, pp. 869-878, 2002.

[14] T. D. Lamkin, S. F. Walk, L. Liu, J. E. Damen, G. Krystal, and K. S. Ravichandran, "Shc interaction with Shc homology 2 domain containing inositol phosphatase (SHIP) in vivo requires the Shc-phosphotyrosine binding domain and two specific phosphotyrosines on SHIP," The Journal of Biological Chemistry, vol. 272, no. 16, pp. 10396-10401, 1997.

[15] H. Chin, T. Saito, A. Arai et al., "Erythropoietin and IL-3 induce tyrosine phosphorylation of CrkL and its association with Shc, SHP-2, and Cbl in hematopoietic cells," Biochemical and Biophysical Research Communications, vol. 239, no. 2, pp. 412-417, 1997.

[16] S. Ni, C. Zhao, G. S. Feng, R. F. Paulson, and P. H. Correll, "A novel stat3 binding motif in Gab2 mediates transformation of primary hematopoietic cells by the Stk/Ron receptor tyrosine kinase in response to friend virus infection," Molecular and Cellular Biology, vol. 27, no. 10, pp. 3708-3715, 2007.
[17] N. Harir, C. Pecquet, M. Kerenyi et al., "Constitutive activation of stat 5 promotes its cytoplasmic localization and association with PI3-kinase in myeloid leukemias," Blood, vol. 109, no. 4, pp. 1678-1686, 2007.

[18] C. Crouin, M. Arnaud, F. Gesbert, J. Camonis, and J. Bertoglio, "A yeast two-hybrid study of human p97/Gab2 interactions with its $\mathrm{SH} 2$ domain-containing binding partners," Febs Letters, vol. 495, no. 3, pp. 148-153, 2001.

[19] M. Arnaud, C. Crouin, C. Deon, D. Loyaux, and J. Bertoglio, "Phosphorylation of Grb2-associated binder 2 on serine 623 by ERK MAPK regulates its association with the phosphatase SHP-2 and decreases STAT5 activation," Journal of Immunology, vol. 173, no. 6, pp. 3962-3971, 2004.

[20] D. K. Lynch and R. J. Daly, "PKB-mediated negative feedback tightly regulates mitogenic signalling via Gab2," The Embo Journal, vol. 21, no. 1-2, pp. 72-82, 2002.

[21] S. Zhang and H. E. Broxmeyer, "Flt3 ligand induces tyrosine phosphorylation of Gab1 and Gab2 and their association with Shp-2, Grb2, and PI3 kinase," Biochemical and Biophysical Research Communications, vol. 277, no. 1, pp. 195-199, 2000.

[22] C. Bourgin, R. P. Bourette, S. Arnaud, Y. Liu, L. R. Rohrschneider, and G. Mouchiroud, "Induced expression and association of the Mona/Gads adapter and Gab3 scaffolding protein during monocyte/macrophage differentiation," Molecular and Cellular Biology, vol. 22, no. 11, pp. 3744-3756, 2002.

[23] K. Carlberg and L. R. Rohrschneider, "Characterization of a novel tyrosine phosphorylated $100-\mathrm{kDa}$ protein that binds to SHP-2 and phosphatidylinositol 3'-kinase in myeloid cells," Journal of Biological Chemistry, vol. 272, no. 25, pp. 1594315950, 1997.

[24] A. W. Lee and D. J. States, "Both src-dependent andindependent mechanisms mediate phosphatidylinositol 3kinase regulation of colony-stimulating factor 1-activated mitogen-activated protein kinases in myeloid progenitors," Molecular and Cellular Biology, vol. 20, no. 18, pp.6779-6798, 2000.

[25] Y. Liu, B. Jenkins, Jun Lim Shin, and L. R. Rohrschneider, "Scaffolding protein Gab2 mediates differentiation signaling downstream of Fms receptor tyrosine kinase," Molecular and Cellular Biology, vol. 21, no. 9, pp. 3047-3056, 2001.

[26] P. D. Simoncic, A. Bourdeau, A. Lee-Loy et al., "T-cell protein tyrosine phosphatase (Tcptp) is a negative regulator of colony-stimulating factor 1 signaling and macrophage differentiation," Molecular and Cellular Biology, vol. 26, no. 11, pp. 4149-4160, 2006.

[27] K. Nishida, L. Wang, E. Morii et al., "Requirement of Gab2 for mast cell development and KitL/c-Kit signaling," Blood, vol. 99, no. 5, pp. 1866-1869, 2002.

[28] M. Yu, J. Luo, W. Yang et al., "The scaffolding adapter Gab2, via Shp-2, regulates Kit-evoked mast cell proliferation by activating the Rac/JNK pathway," The Journal of Biological Chemistry, vol. 281, no. 39, pp. 28615-28626, 2006.

[29] K. Nishida, Y. Yoshida, M. Itoh et al., "Gab-family adapter proteins act downstream of cytokine and growth factor receptors and T-and B-cell antigen receptors," Blood, vol. 93, no. 6, pp. 1809-1816, 1999.

[30] S. Takaki, H. Morita, Y. Tezuka, and K. Takatsu, "Enhanced hematopoiesis by hematopoietic progenitor cells lacking intracellular adaptor protein, Lnk," The Journal of Experimental Medicine, vol. 195, no. 2, pp. 151-160, 2002. 
[31] A. K. Gandhi, J. Kang, S. Naziruddin, A. Parton, P. H. Schafer, and D. I. Stirling, "Lenalidomide inhibits proliferation of namalwa CSN.70 cells and interferes with Gabl phosphorylation and adaptor protein complex assembly," Leukemia Research, vol. 30, no. 7, pp. 849-858, 2006.

[32] K. Nishigaki, C. Hanson, T. Ohashi, D. Thompson, K. Muszynski, and S. Ruscetti, "Erythroid cells rendered erythropoietin independent by infection with friend spleen focus-forming virus show constitutive activation of phosphatidylinositol 3-kinase and Akt kinase: involvement of insulin receptor substrate-related adapter proteins," Journal of Virology, vol. 74, no. 7, pp. 3037-3045, 2000.

[33] E. van den Akker, T. van Dijk, M. Parren-Van Amelsvoort et al., "Tyrosine kinase receptor RON functions downstream of the erythropoietin receptor to induce expansion of erythroid progenitors," Blood, vol. 103, no. 12, pp. 4457-4465, 2004.

[34] D. Bouscary, F. Pene, Y. E. Claessens et al., "Critical role for PI 3-kinase in the control of erythropoietin-induced erythroid progenitor proliferation," Blood, vol. 101, no. 9, pp. 34363443, 2003.

[35] Q. S. Zhu, L. J. Robinson, V. Roginskaya, and S. J. Corey, "G-CSF-induced tyrosine phosphorylation of Gab2 is Lyn kinase dependent and associated with enhanced Akt and differentiative, not proliferative, responses," Blood, vol. 103, no. 9, pp. 3305-3312, 2004.

[36] H. Gu, H. Maeda, J. J. Moon et al., "New role for Shc in activation of the phosphatidylinositol 3-kinase/Akt pathway," Molecular and Cellular Biology, vol. 20, no. 19, pp. 7109-7120, 2000.

[37] H. Gu, J. D. Griffin, and B. G. Neel, "Characterization of two SHP-2-associated binding proteins and potential substrates in hematopoietic cells," The Journal of Biological Chemistry, vol. 272, no. 26, pp. 16421-16430, 1997.

[38] C. Lecoq-Lafon, F. Verdier, S. Fichelson et al., "Erythropoietin induces the tyrosine phosphorylation of GAB1 and its association with SHC, SHP2, SHIP, and phosphatidylinositol 3-kinase," Blood, vol. 93, no. 8, pp. 2578-2585, 1999.

[39] M. Takahashi-Tezuka, Y. Yoshida, T. Fukada et al., "Gab1 acts as an adapter molecule linking the cytokine receptor gp130 to ERK mitogen-activated protein kinase," Molecular and Cellular Biology, vol. 18, no. 7, pp. 4109-4117, 1998.

[40] K. Podar, G. Mostoslavsky, M. Sattler et al., "Critical role for hematopoietic cell kinase (Hck)-mediated phosphorylation of Gab1 and Gab2 docking proteins in interleukin 6-induced proliferation and survival of multiple myeloma cells," The Journal of Biological Chemistry, vol. 279, no. 20, pp. 2165821665, 2004.

[41] A. Badache and N. E. Hynes, "Interleukin 6 inhibits proliferation and, in cooperation with an epidermal growth factor receptor autocrine loop, increases migration of T47D breast cancer cells," Cancer Research, vol. 61, no. 1, pp. 383-391, 2001.

[42] T. Fukada, M. Hibi, Y. Yamanaka et al., "Two signals are necessary for cell proliferation induced by a cytokine receptor gp130: involvement of STAT3 in anti-apoptosis," Immunity, vol. 5, no. 5, pp. 449-460, 1996.

[43] Y. Nakaoka, K. Nishida, Y. Fujio et al., "Activation of gp130 transduces hypertrophic signal through interaction of scaffolding/docking protein Gabl with tyrosine phosphatase SHP2 in cardiomyocytes," Circulation Research, vol. 93, no. 3, pp. 221-229, 2003.

[44] J. L. Brockdorff, H. Gu, T. Mustelin et al., "Gab2 is phosphorylated on tyrosine upon interleukin-2/interleukin-15 stimulation in mycosis-fungoides-derived tumor T cells and associates inducibly with SHP-2 and stat5a," Experimental and Clinical Immunogenetics, vol. 18, no. 2, pp. 86-95, 2001.

[45] M. Gadina, C. Sudarshan, R. Visconti et al., "The docking molecule Gab2 is induced by lymphocyte activation and is involved in signaling by interleukin-2 and interleukin-15 but not other common $\gamma$ chain-using cytokines," The Journal of Biological Chemistry, vol. 275, no. 35, pp. 26959-26966, 2000.

[46] M. Gadina, C. Sudarshan, and J. J. O'Shea, "IL-2, but not IL-4 and other cytokines, induces phosphorylation of a 98$\mathrm{kDa}$ protein associated with SHP-2, phosphatidylinositol 3'kinase, and Grb2," Journal of Immunology, vol. 162, no. 4, pp. 2081-2086, 1999.

[47] F. Gesbert, C. Guenzi, and J. Bertoglio, "A new tyrosinephosphorylated $97-\mathrm{kDa}$ adaptor protein mediates interleukin-2-induced association of SHP-2 with p85-phosphatidylinositol 3- kinase in human T lymphocytes," The Journal of Biological Chemistry, vol. 273, no. 29, pp. 18273-18281, 1998.

[48] W. M. Yu, T. S. Hawley, R. G. Hawley, and C. K. Qu, "Role of the docking protein Gab2 in $\beta 1$-integrin signaling pathwaymediated hematopoietic cell adhesion and migration," Blood, vol. 99, no. 7, pp. 2351-2359, 2002.

[49] B. L. Craddock, J. Hobbs, C. E. Edmead, and M. J. Welham, "Phosphoinositide 3-kinase-dependent regulation of interleukin-3-induced proliferation: involvement of mitogen-activated protein kinases, SHP2 and Gab2," The Journal of Biological Chemistry, vol. 276, no. 26, pp. 24274-24283, 2001.

[50] B. L. Craddock, E. A. Orchiston, H. J. Hinton, and M. J. Welham, "Dissociation of apoptosis from proliferation, protein kinase $\mathrm{B}$ activation, and $\mathrm{BAD}$ phosphorylation in interleukin-3-mediated phosphoinositide 3-kinase signaling," Journal of Biological Chemistry, vol. 274, no. 15, pp. 10633-10640, 1999.

[51] B. L. Craddock and M. J. Welham, "Interleukin-3 induces association of the protein-tyrosine phosphatase SHP2 and phosphatidylinositol 3-kinase with a 100-kDa tyrosinephosphorylated protein in hemopoietic cells," The Journal of Biological Chemistry, vol. 272, no. 46, pp. 29281-29289, 1997.

[52] W. M. Yu, T. S. Hawley, R. G. Hawley, and C. K. Qu, "Catalytic-dependent and -independent roles of SHP-2 tyrosine phosphatase in interleukin-3 signaling," Oncogene, vol. 22, no. 38, pp. 5995-6004, 2003.

[53] W. J. Tao, H. Lin, T. Sun, A. K. Samanta, and R. Arlinghaus, "BCR-ABL oncogenic transformation of NIH 3T3 fibroblasts requires the IL-3 receptor," Oncogene, vol. 27, no. 22, pp. 3194-3200, 2008.

[54] Y. Miyakawa, P. Rojnuckarin, T. Habib, and K. Kaushansky, "Thrombopoietin induces phosphoinositol 3-kinase activation through SHP2, Gab, and insulin receptor substrate proteins in BAF3 cells and primary murine megakaryocytes," The Journal of Biological Chemistry, vol. 276, no. 4, pp. 24942502, 2001.

[55] D. Bouscary, C. Lecoq-Lafon, S. Chretien et al., "Role of Gab proteins in phosphatidylinositol 3-kinase activation by thrombopoietin (Tpo)," Oncogene, vol. 20, no. 18, pp. 21972204, 2001.

[56] H. Kojima, A. Shinagawa, S. Shimizu et al., "Role of phosphatidylinositol-3 kinase and its association with Gab1 in thrombopoietin-mediated up-regulation of platelet function," Experimental Hematology, vol. 29, no. 5, pp. 616-622, 2001.

[57] P. Rojnuckarin, Y. Miyakawa, N. E. Fox, J. Deou, G. Daum, and K. Kaushansky, "The roles of phosphatidylinositol 3kinase and protein kinase $\mathrm{C} \zeta$ for thrombopoietin-induced 
mitogen-activated protein kinase activation in primary murine megakaryocytes," Journal of Biological Chemistry, vol. 276, no. 44, pp. 41014-41022, 2001.

[58] M. Kong, C. Mounier, J. Wu, and B. I. Posner, "Epidermal growth factor-induced phosphatidylinositol 3-kinase activation and DNA synthesis: identification of Grb2-associated binder 2 as the major mediator in rat hepatocytes," Journal of Biological Chemistry, vol. 275, no. 46, pp. 36035-36042, 2000.

[59] M. Kong, C. Mounier, A. Balbis, G. Baquiran, and B. I. Posner, "Gab2 tyrosine phosphorylation by a pleckstrin homology domain-independent mechanism: role in epidermal growth factor-induced mitogenesis," Molecular Endocrinology, vol. 17, no. 5, pp. 935-944, 2003.

[60] J. Sun, M. Pedersen, and L. Ronnstrand, "Gab2 is involved in differential phosphoinositide 3-kinase signaling by two splice forms of c-Kit," Journal of Biological Chemistry, vol. 283, no. 41, pp. 27444-27451, 2008.

[61] H. Yoshihara, F. Arai, K. Hosokawa et al., "Thrombopoietin/MPL signaling regulates hematopoietic stem cell quiescence and interaction with the osteoblastic niche," Cell Stem Cell, vol. 1, no. 6, pp. 685-697, 2007.

[62] M. Itoh, Y. Yoshida, K. Nishida, M. Narimatsu, M. Hibi, and T. Hirano, "Role of Gab l in heart, placenta, and skin development and growth factor-and cytokine-induced extracellular signal-regulated kinase mitogen-activated protein kinase activation," Molecular and Cellular Biology, vol. 20, no. 10, pp. 3695-3704, 2000.

[63] M. Sachs, H. Brohmann, D. Zechner et al., "Essential role of Gab1 for signaling by the c-Met receptor in vivo," The Journal of Cell Biology, vol. 150, no. 6, pp. 1375-1384, 2000.

[64] D. W. Threadgill, A. A. Dlugosz, L. A. Hansen et al., "Targeted disruption of mouse EGF receptor: effect of genetic background on mutant phenotype," Science, vol. 269, no. 5221, pp. 230-234, 1995.

[65] L. Karlsson, C. Bondjers, and C. Betsholtz, "Roles for PDGFA and sonic hedgehog in development of mesenchymal components of the hair follicle," Development, vol. 126, no. 12, pp. 2611-2621, 1999.

[66] P. Leveen, M. Pekny, S. Gebre-Medhin, B. Swolin, E. Larsson, and C. Betsholtz, "Mice deficient for PDGF B show renal, cardiovascular, and hematological abnormalities," Genes \& Development, vol. 8, no. 16, pp. 1875-1887, 1994.

[67] F. Bladt, D. Riethmacher, S. Isenmann, A. Aguzzi, and C. Birchmeier, "Essential role for the c-met receptor in the migration of myogenic precursor cells into the limb bud," Nature, vol. 376, no. 6543, pp. 768-771, 1995.

[68] C. Schmidt, F. Bladt, S. Goedecke et al., "Scatter factor/hepatocyte growth factor is essential for liver development," Nature, vol. 373, no. 6516, pp. 699-702, 1995.

[69] R. Ohlsson, P. Falck, M. Hellstrom et al., "PDGFB regulates the development of the labyrinthine layer of the mouse fetal placenta," Developmental Biology, vol. 212, no. 1, pp. 124136, 1999.

[70] C. K. Qu, W. M. Yu, B. Azzarelli, S. Cooper, H. E. Broxmeyer, and G. S. Feng, "Biased suppression of hematopoiesis and multiple developmental defects in chimeric mice containing Shp-2 mutant cells," Molecular and Cellular Biology, vol. 18, no. 10, pp. 6075-6082, 1998.

[71] E. A. Bard-Chapeau, A. L. Hevener, S. Long, E. E. Zhang, J. M. Olefsky, and G. S. Feng, "Deletion of Gab1 in the liver leads to enhanced glucose tolerance and improved hepatic insulin action," Nature Medicine, vol. 11, no. 5, pp. 567-571, 2005.
[72] H. Gu, K. Saito, L. D. Klaman et al., "Essential role for Gab2 in the allergic response," Nature, vol. 412, no. 6843, pp. 186190, 2001.

[73] C. P. Shelburne, M. E. McCoy, R. Piekorz et al., "Stat5 expression is critical for mast cell development and survival," Blood, vol. 102, no. 4, pp. 1290-1297, 2003.

[74] C. E. Edmead, B. C. Fox, C. Stace, N. Ktistakis, and M. J. Welham, "The pleckstrin homology domain of Gab-2 is required for optimal interleukin-3 signalsome-mediated responses," Cellular Signalling, vol. 18, no. 8, pp. 1147-1155, 2006.

[75] K. Nishida, S. Yamasaki, A. Hasegawa et al., "Gab2, via PI$3 \mathrm{~K}$, regulates ARF1 in fcepsilonRI-mediated granule translocation and mast cell degranulation," Journal of Immunology, vol. 187, no. 2, pp. 932-941, 2011.

[76] Y. Zhang, E. Diaz-Flores, G. Li et al., "Abnormal hematopoiesis in Gab2 mutant mice," Blood, vol. 110, no. 1, pp. 116-124, 2007.

[77] R. P. Bourette, S. Arnaud, G. M. Myles, J. P. Blanchet, L. R. Rohrschneider, and G. Mouchiroud, "Mona, a novel hematopoietic-specific adaptor interacting with the macrophage colony-stimulating factor receptor, is implicated in monocyte/macrophage development," The Embo Journal, vol. 17, no. 24, pp. 7273-7281, 1998.

[78] M. Seiffert, J. M. Custodio, I. Wolf et al., "Gab3-deficient mice exhibit normal development and hematopoiesis and are immunocompetent," Molecular and Cellular Biology, vol. 23, no. 7, pp. 2415-2424, 2003.

[79] R. J. Daly, H. Gu, J. Parmar et al., “The docking protein Gab2 is overexpressed and estrogen regulated in human breast cancer," Oncogene, vol. 21, no. 33, pp. 5175-5181, 2002.

[80] M. Bentires-Alj, S. G. Gil, R. Chan et al., "A role for the scaffolding adapter GAB2 in breast cancer," Nature Medicine, vol. 12, no. 1, pp. 114-121, 2006.

[81] T. Brummer, D. Schramek, V. M. Hayes et al., "Increased proliferation and altered growth factor dependence of human mammary epithelial cells overexpressing the Gab2 docking protein," The Journal of Biological Chemistry, vol. 281, no. 1, pp. 626-637, 2006.

[82] Y. Ke, D. Wu, F. Princen et al., "Role of Gab2 in mammary tumorigenesis and metastasis," Oncogene, vol. 26, no. 34, pp. 4951-4960, 2007.

[83] H. L. Bennett, T. Brummer, A. Jeanes, A. S. Yap, and R. J. Daly, "Gab2 and src co-operate in human mammary epithelial cells to promote growth factor independence and disruption of acinar morphogenesis," Oncogene, vol. 27, no. 19, pp. 26932704, 2008.

[84] J. M. Stommel, A. C. Kimmelman, H. Ying et al., "Coactivation of receptor tyrosine kinases affects the response of tumor cells to targeted therapies," Science, vol. 318, no. 5848, pp. 287-290, 2007.

[85] V. de Falco, V. Guarino, L. Malorni et al., "RAI(ShcC/NShc)-dependent recruitment of GAB1 to RET oncoproteins potentiates $\mathrm{PI} 3-\mathrm{K}$ signalling in thyroid tumors," Oncogene, vol. 24, no. 41, pp. 6303-6313, 2005.

[86] S. H. Lee, E. G. Jeong, S. W. Nam, J. Y. Lee, N. J. Yoo, and S. $\mathrm{H}$. Lee, "Increased expression of Gab2, a scaffolding adaptor of the tyrosine kinase signalling, in gastric carcinomas," Pathology, vol. 39, no. 3, pp. 326-329, 2007.

[87] M. Sattler, M. G. Mohi, Y. B. Pride et al., "Critical role for Gab2 in transformation by BCR/ABL," Cancer Cell, vol. 1, no. 5, pp. 479-492, 2002. 
[88] H. E. Teal, S. Ni, J. Xu et al., "GRB2-mediated recruitment of GAB2, but not GAB1, to SF-STK supports the expansion of friend virus-infected erythroid progenitor cells," Oncogene, vol. 25, no. 17, pp. 2433-2443, 2006.

[89] C. Voena, C. Conte, C. Ambrogio et al., "The tyrosine phosphatase Shp2 interacts with NPM-ALK and regulates anaplastic lymphoma cell growth and migration," Cancer Research, vol. 67, no. 9, pp. 4278-4286, 2007.

[90] K. Masson, T. Liu, R. Khan, J. Sun, and L. Ronnstrand, "A role of Gab2 association in Flt3 ITD mediated stat5 phosphorylation and cell survival," British Journal of Haematology, vol. 146, no. 2, pp. 193-202, 2009.

[91] A. Zatkova, C. Schoch, F. Speleman et al., "GAB2 is a novel target of $11 \mathrm{q}$ amplification in AML/MDS," Genes Chromosomes \& Cancer, vol. 45, no. 9, pp. 798-807, 2006.

[92] V. M. Zaleskas, D. S. Krause, K. Lazarides et al., "Molecular pathogenesis and therapy of polycythemia induced in mice by JAK2 V617F," Plos One, vol. 1, no. 1, article e18, 2006.

[93] K. Mood, C. Saucier, Y. S. Bong, H. S. Lee, M. Park, and I. O. Daar, "Gabl is required for cell cycle transition, cell proliferation, and transformation induced by an oncogenic met receptor," Molecular Biology of the Cell, vol. 17, no. 9, pp. 3717-3728, 2006.

[94] M. Holgado-Madruga and A. J. Wong, "Role of the Grb2associated binder 1/SHP-2 interaction in cell growth and transformation," Cancer Research, vol. 64, no. 6, pp. 20072015, 2004.

[95] A. Felici, A. Giubellino, and D. P. Bottaro, "Gab1 mediates hepatocyte growth factor-stimulated mitogenicity and morphogenesis in multipotent myeloid cells," Journal of Cellular Biochemistry, vol. 111, no. 2, pp. 310-321, 2010.

[96] S. Roumiantsev, D. S. Krause, C. A. Neumann et al., "Distinct stem cell myeloproliferative/T lymphoma syndromes induced by ZNF198-FGFR1 and BCR-FGFR1 fusion genes from 8p11 translocations," Cancer Cell, vol. 5, no. 3, pp. 287298, 2004.

[97] M. Scherr, A. Chaturvedi, K. Battmer et al., "Enhanced sensitivity to inhibition of SHP2, STAT5, and Gab2 expression in chronic myeloid leukemia (CML)," Blood, vol. 107, no. 8, pp. 3279-3287, 2006.

[98] M. H. Nguyen, J. M. Ho, B. K. Beattie, and D. L. Barber, "TEL-JAK2 mediates constitutive activation of the phosphatidylinositol 3'-kinase/protein kinase B signaling pathway," The Journal of Biological Chemistry, vol. 276, no. 35, pp. 32704-32713, 2001.

[99] R. P. Million, N. Harakawa, S. Roumiantsev, L. Varticovski, and R. A. Van Etten, "A direct binding site for Grb2 contributes to transformation and leukemogenesis by the Tel-Abl (ETV6-Abl) tyrosine kinase," Molecular and Cellular Biology, vol. 24, no. 11, pp. 4685-4695, 2004.

[100] D. Xu, S. Wang, W. M. Yu et al., "A germline gain-of-function mutation in Ptpn11 (Shp-2) phosphatase induces myeloproliferative disease by aberrant activation of hematopoietic stem cells," Blood, vol. 116, no. 18, pp. 3611-3621, 2010.

[101] Y. Yuan, L. Qin, D. Liu et al., "Genetic screening reveals an essential role of p27kip1 in restriction of breast cancer progression," Cancer Research, vol. 67, no. 17, pp. 8032-8042, 2007.

[102] M. T. Abreu, W. E. Hughes, K. Mele et al., "Gab2 regulates cytoskeletal organization and migration of mammary epithelial cells by modulating RhoA activation," Molecular Biology of the Cell, vol. 22, no. 1, pp. 105-116, 2011.
[103] K. Abe, K. L. Rossman, B. Liu et al., "Vav2 is an activator of Cdc42, Rac1, and RhoA," The Journal of Biological Chemistry, vol. 275, no. 14, pp. 10141-10149, 2000.

[104] F. Bernal, M. Wade, M. Godes et al., "A stapled p53 helix overcomes HDMX-mediated suppression of p53," Cancer Cell, vol. 18, no. 5, pp. 411-422, 2010.

[105] C. R. Braun, J. Mintseris, E. Gavathiotis, G. H. Bird, S. P. Gygi, and L. D. Walensky, "Photoreactive stapled $\mathrm{BH} 3$ peptides to dissect the BCL-2 family interactome," Chemistry \& Biology, vol. 17, no. 12, pp. 1325-1333, 2010. 


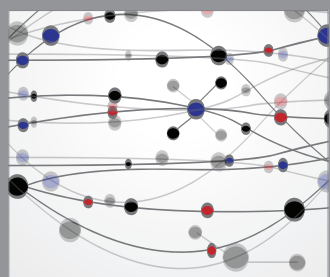

The Scientific World Journal
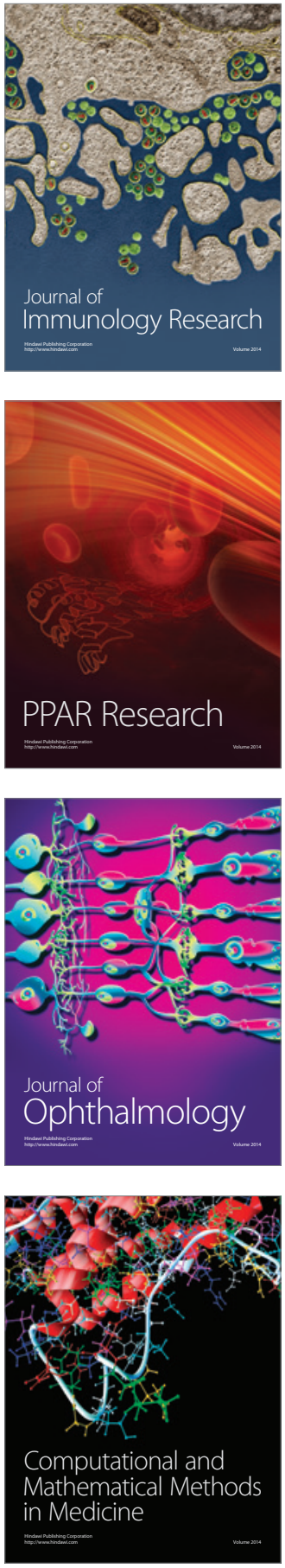

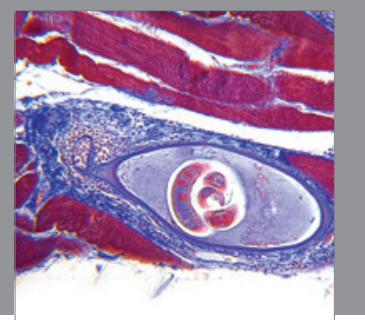

Gastroenterology

Research and Practice
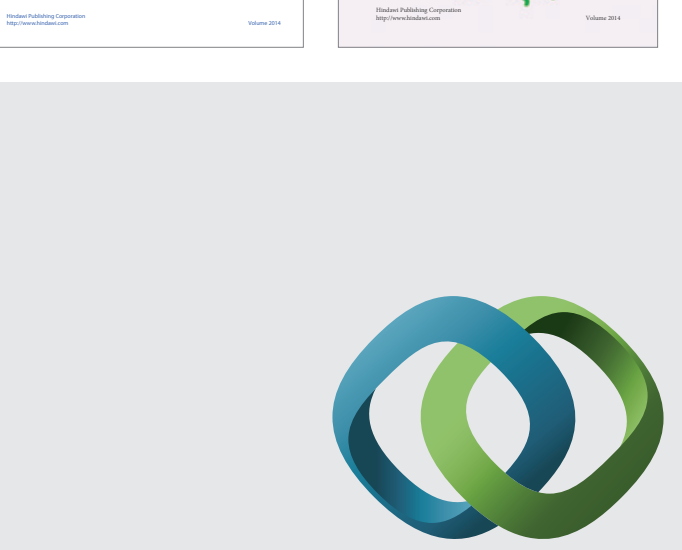

\section{Hindawi}

Submit your manuscripts at

http://www.hindawi.com
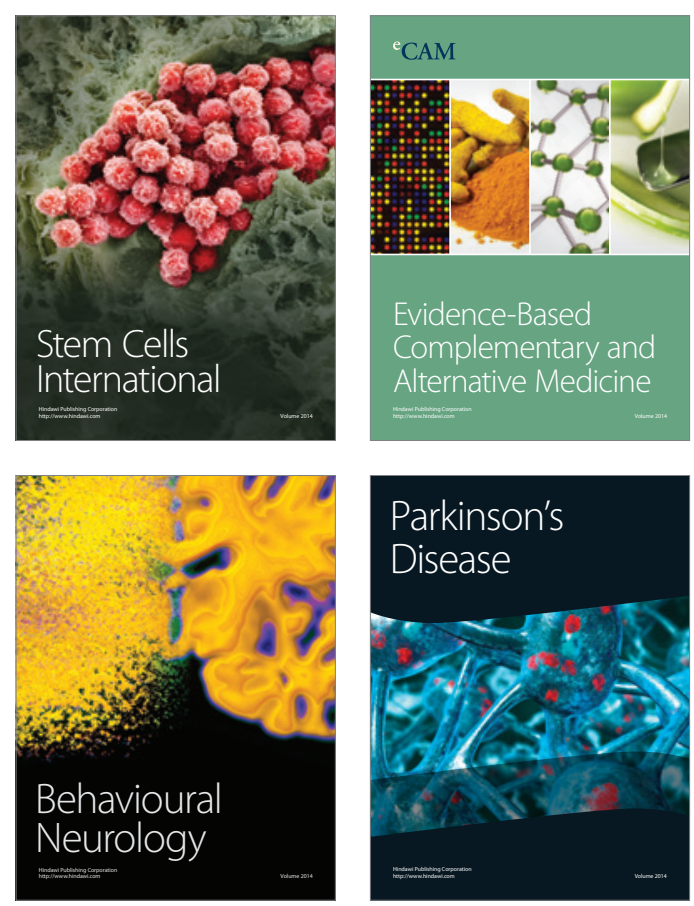

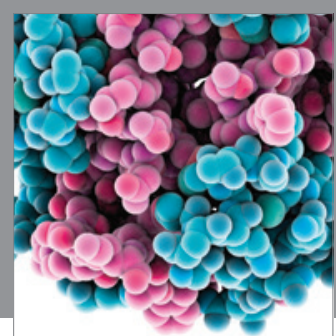

Journal of
Diabetes Research

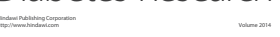

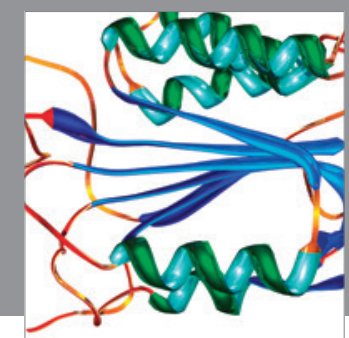

Disease Markers
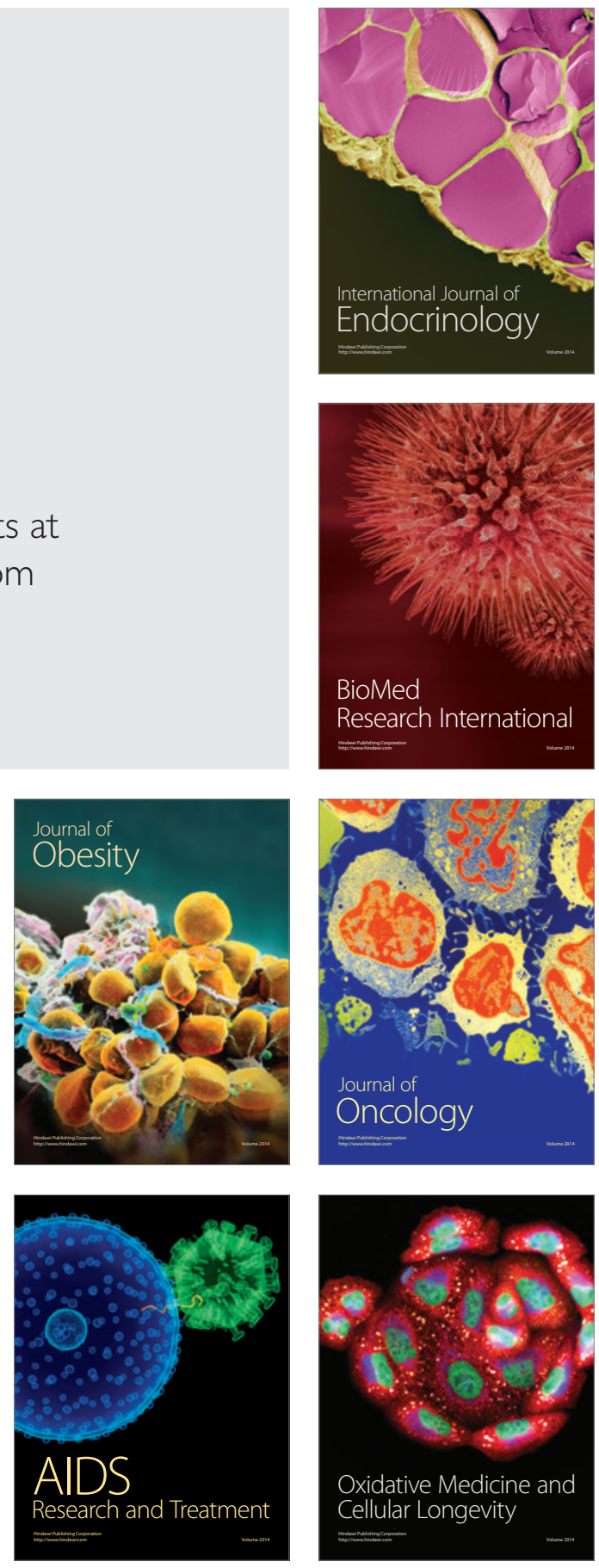